\title{
Metformin ameliorates bile duct ligation-induced acute hepatic injury via regulation of ER stress
}

\author{
Chi-Ho Lee ${ }^{1, \#}$, Jung-Hwa Han ${ }^{1, \#}$, Sujin Kim ${ }^{1}$, Heejung Lee ${ }^{1}$, Suji Kim ${ }^{1}$, Dae-Hwan Nam ${ }^{2}$, Du-Hyong Cho $^{1}$ \& \\ Chang-Hoon Woo ${ }^{\text {,** }}$ \\ ${ }^{1}$ Department of Pharmacology and Smart-Ageing Convergence Research Center, Yeungnam University College of Medicine, Daegu 42415, \\ Korea, ${ }^{2}$ Center for Inflammation, Immunity \& Infection, Institute for Biomedical Sciences, Georgia State University, Atlanta, GA 30302, \\ USA
}

\begin{abstract}
Cholestasis is a condition in which the bile duct becomes narrowed or clogged by a variety of factors and bile acid is not released smoothly. Bile acid-induced liver injury is facilitated by necrotic cell death, neutrophil infiltration, and inflammation. Metformin, the first-line treatment for type 2 diabetes, is known to reduce not only blood glucose but also inflammatory responses. In this study, we investigated the effects of metformin on liver injury caused by cholestasis with bile acid-induced hepatocyte injury. Static bile acid-induced liver injury is thought to be related to endoplasmic reticulum (ER) stress, inflammatory response, and chemokine expression. Metformin treatment reduced liver injury caused by bile acid, and it suppressed ER stress, inflammation, chemokine expression, and neutrophil infiltration. Similar results were obtained in mouse primary hepatocytes exposed to bile acid. Hepatocytes treated with tauroursodeoxycholic acid, an ER stress inhibitor, showed inhibition of ER stress, as well as reduced levels of inflammation and cell death. These results suggest that metformin may protect against liver injury by suppressing ER stress and inflammation and reducing chemokine expression. [BMB Reports 2020; 53(6): 311-316]
\end{abstract}

\section{INTRODUCTION}

Metformin is known as the first-line treatment for type 2 diabetes, as it is highly effective in lowering blood glucose levels and has few side effects (1). Metformin acts on hepatocytes to inhibit mitochondrial complex 1 . As a result, the concentration

*Corresponding author. Tel: +82-53-640-6973; Fax: +82-53-656-7995; E-mail: changhoon_woo@yu.ac.kr

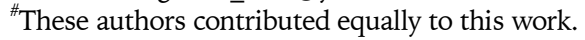

https://doi.org/10.5483/BMBRep.2020.53.6.169

Received 8 July 2019, Revised 5 August 2019,

Accepted 13 November 2019

Keywords: Bile acid, ER stress, Hepatic injury, Metformin, Unfolded protein response of adenosine monophosphate (AMP) in the cytoplasm is increased, activating protein kinase A (PKA) and AMP-activated protein kinase (AMPK). Activation of AMPK inhibits lipogenesis, whereas AMP and some downstream targets of PKA inhibit gluconeogenesis (2). Metformin is known to have not only a therapeutic effect on diabetes mellitus but also several other beneficial effects including anti-inflammatory (3) and antioxidative (4) effects. It is also reported to have an anticancer effect (5) and an endoplasmic reticulum (ER) stress-reducing effect (6).

Cholestasis is a condition in which bile acid is not discharged smoothly indicative of a bile duct that has narrowed or become blocked by various factors. These factors include genetic defects, adverse drug reactions, and gallstones $(7,8)$. Cholestasis may result in the stasis of bile acids, and liver injury may result from bile acid stagnation (8). In vitro, hepatocyte apoptosis has been reported to be caused by bile acid (9). In a mouse model of cholestasis with bile duct ligation (BDL), necrotic core is one of key histological features of liver injury and leads neutrophil infiltration. Necrotic core could be caused by mixed types of cell death including necrosis and apoptosis. It has been reported that cholestasis in a mouse model of BDL revealed ER stress (10) and inflammatory (11) responses after $\mathrm{BDL}$, resulting in the release of chemokines and calls for neutrophils to approach hepatocytes. Such approaches of neutrophils to hepatocytes have been reported to necrotize hepatocytes (9). However, the intermediate pathway by which necrotic hepatic injury is regulated by necroptosis and ER stress has not been described.

ER stress occurs when unfolded or misfolded proteins are present inside the ER, which is when the homeostasis of cells, such as that associated with oxidative stress or inflammation, is not properly maintained. When ER stress occurs, cells work to normalize cell functions, such as suppressing mRNA translation, improving the folding ability of proteins, and dispensing unnecessary proteins, in order to maintain homeostasis. Under chronic or unresolved ER stress, cells undergo degeneration that can result in apoptosis (12). Recent reports have showed that ER stress is closely involved in the regulation of hepatic injury and cell death of vascular smooth muscle cells $(13,14)$.

ISSN: 1976-670X (electronic edition)

Copyright (c) 2020 by the The Korean Society for Biochemistry and Molecular Biology

(c) This is an open-access article distributed under the terms of the Creative Commons Attribution Non-Commercial License (http://creativecommons.org/licenses/by-nc/4.0) which permits unrestricted non-commercial use, distribution, and reproduction in any medium, provided the original work is properly cited. 
It has been reported that metformin can inhibit ER stress and suppress unfolded protein responses to bile acid $(6,15)$.

In this study, we investigated the effect of metformin on liver injury caused by the stagnation of bile and examined the pathway through which the metformin effect was induced in a BDL mouse model of cholestasis.

\section{RESULTS AND DISCUSSION}

\section{Metformin recovers bile duct ligation-induced hepatic injury, chemokine expression, and inflammation}

It has been reported that an experimental model for the induction of obstructive cholestatic injury in mice can be obtained through surgical bile duct ligation (BDL) (16). Previous reports have shown that inflammatory responses are prominent from 3 and 5 days after BDL surgery but recover after 7 days, and liver fibrosis occurs at 14 days after BDL surgery (17). To examine the effect of metformin on cholestasis, C57BL/6 mice were subjected to BDL for 3 days with or without metformin administration. The gross appearance of the livers from the metformintreated group was remarkably normal compared with that of the untreated group (Fig. 1A). Since necrotic core is one of key histological features of liver injury induced by BDL, we assessed necrotic area by comparatively examining H\&E staining of liver sections from mice. As shown in Fig. 1B, BDL-operated mice displayed increased focal necrosis (infarct) and portal inflammation, changes that are consistent with duct obstruction. In metformin-treated BDL-operated mice, the areas of infarcts were significantly reduced compared to non-metformin-

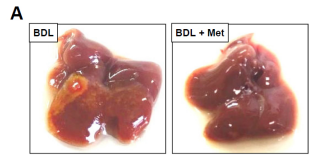

B

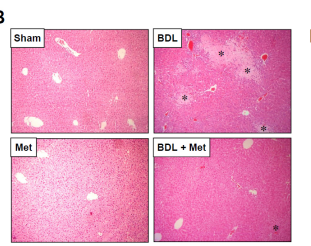

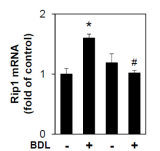

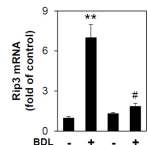
$\underset{80}{10}+2$<smiles></smiles>

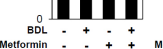

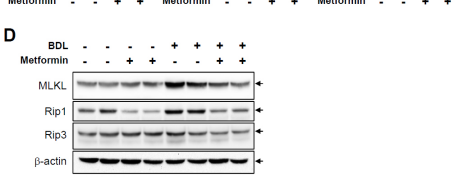

Fig. 1. Metformin reduces bile duct ligation-induced hepatic injury and necroptosis. Sham or BDL-operated mice were injected with metformin (i.p, $200 \mathrm{mg} / \mathrm{kg} /$ day) for 3 days, and then liver tissues were harvested ( $n=5$ ). (A) Representative pictures of whole livers from treated mice. (B) Liver tissue sections were subjected to $H \& E$ staining (original magnification, $\times 100$ ). ${ }^{*}$ indicates necrotic area. (C) mRNA levels of MLKL, Rip1, and Rip3 were measured by performing qRT-PCR. Relative expression levels were normalized to GAPDH. Results are expressed as mean \pm SD values and are representative of three independent experiments. ${ }^{*} \mathrm{P}<0.05$ and $* * P<0.01$ vs. Sham, ${ }^{\#} P<0.05$ and ${ }^{\# \#} P<0.01$ vs. BDL. (D) Protein amounts of MLKL, Rip1, Rip3, and $\beta$-actin were determined by immunoblotting with specific antibodies, respectively. BDL: bile duct ligation, Met: metformin, Sham: sham-operated. treated BDL-operated mice. It has been reported that necroptosis is activated in the liver of human and experimental cholestasis (18). To evaluate the effect of metformin in BDLinduced necroptosis, we examined various regulators of necroptosis by determining mixed-lineage kinase domain-like protein (MLKL), receptor-interacting protein1 (Rip1), and receptorinteracting protein3 (Rip3) expression levels. The results showed that BDL-induced MLKL, Rip1, and Rip3 mRNA levels were significantly inhibited by metformin administration (Fig. 1C). In addition, protein levels of necroptic markers are also markedly inhibited by metformin treatment (Fig. 1D), suggesting the protective effects of metformin in BDL-induced hepatic injury.

When bile stagnates, inflammation is caused by the bile, which subsequently induces the secretion of chemokines. It has been reported that neutrophil infiltration in liver tissue induced by secreted chemokines can result in liver tissue damage (9). An immunohistochemical technique was applied to examine neutrophil infiltration in liver tissue by using a specific antibody to mouse neutrophils. A marked increase in neutrophil infiltration was observed in BDL-operated mice, and the neutrophil induction in BDL-operated mice was inhibited by metformin administration (Fig. 2A). Furthermore, BDLinduced CXCL2 and CXCL10 mRNA levels were inhibited by metformin administration (Fig. 2B). Since it has been reported that inflammatory factors can be increased by BDL, we investigated whether metformin could affect inflammation. Circulating $\mathrm{TNF}-\alpha$ in plasma was significantly reduced by metformin
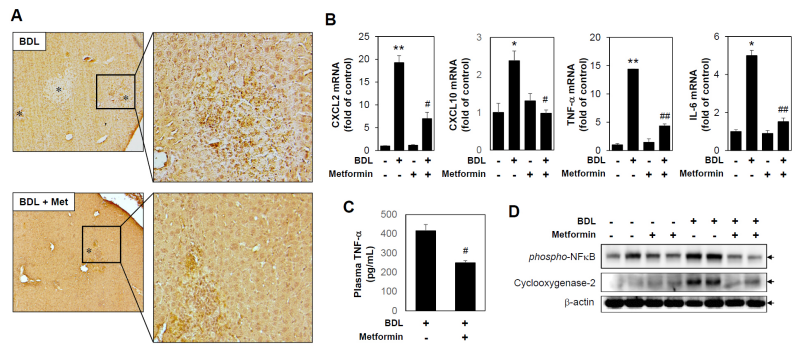

Fig. 2. Metformin reduces bile duct ligation-induced neutrophil infiltration and inflammation. Sham or BDL-operated mice were injected with metformin (oral garbage, $200 \mathrm{mg} / \mathrm{kg} /$ day) for 3 days, and then liver tissues were harvested $(n=5)$. (A) Immunohistochemical staining of neutrophils in a liver tissue section (original magnification, $\times 100$ ). * indicates necrotic area. (B) mRNA levels of CXCL2, CXCL10, TNF- $\alpha$, and IL- 6 were measured by performing qRT-PCR. Relative expression levels were normalized to GAPDH. Results are expressed as mean \pm SD values and are representative of three independent experiments. $* \mathrm{P}<0.05$ and $* * \mathrm{P}<0.01$ vs. Sham, ${ }^{\#} \mathrm{P}<0.05$ and ${ }^{\# \#} \mathrm{P}<0.01$ vs. BDL. (C) Amounts of plasma TNF- $\alpha$ were determined by ELISA assay. Results are expressed as mean \pm SE values and are representative of three independent experiments. ${ }^{\#} \mathrm{P}<0.05$ vs. BDL. (D) Protein levels were measured by immunoblotting with antibodies against phosphoNFкB, Cyclooxygenase-2 and $\beta$-actin. BDL: bile duct ligation, Met: metformin. 
treatment (Fig. 2C). In addition, BDL-induced TNF- $\alpha$ and IL-6 mRNA levels and phospho-NFKB and cyclooxygenase-2 protein levels from liver tissues, which were significantly inhibited by metformin administration (Fig. 2B and 2D). These results suggest that metformin can inhibit early liver damage induced by cholestasis by reducing chemokine expression, inflammation, and necroptosis in vivo.

\section{Metformin inhibits the bile duct ligation-induced unfolded protein response}

ER stress is known to be a cellular reaction occurring during the early stage of cellular damage, and notable inflammatory responses are seen between 3 and 5 days after BDL surgery, suggesting that the two are closely related. To address whether BDL could induce ER stress, major signaling pathways associated with unfolded protein responses (UPR) were assessed by performing immunoblotting and qPCR against each marker protein. BDL induced protein expressions of GRP78/94, IRE1 $\alpha$, ATF4, and CHOP, and mRNA levels of ATF4, CHOP, and spliced XBP-1, and these inductions were markedly diminished by metformin administration (Fig. 3). These results suggest that metformin can inhibit UPR induced by cholestasis in vivo.

To study the pathophysiological process of acute bile acidinduced liver injury, there are two major experimental models of cholestasis. One is surgical technique with direct ligation of bile duct which caused acute and severe liver injury. The other is a chemical-induced intrahepatic cholestasis with $\alpha$-naphthylisothiocyanate (ANIT) which induce biliary epithelial cell hyper-

A

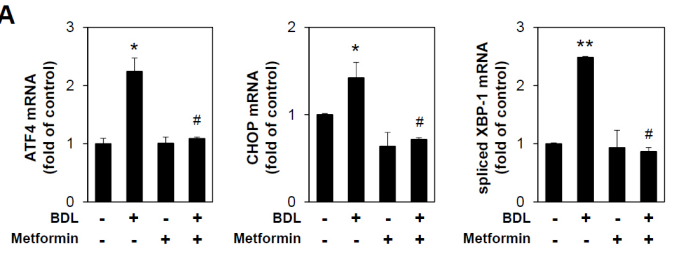

B

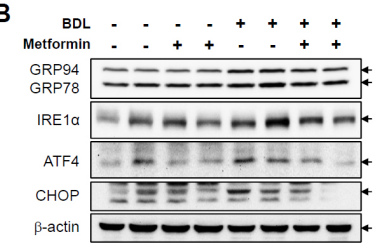

Fig. 3. Metformin inhibits bile duct ligation-induced unfolded protein responses. Sham or BDL-operated mice were injected with metformin (200 mg/kg/day) for 3 days, and then liver tissues were harvested ( $\mathrm{n}$ = 5). (A) mRNA levels of ATF4, CHOP and XBP1s were measured by performing qRT-PCR. Relative expression levels were normalized to GAPDH. Results are expressed as mean \pm SD values and are representative of three independent experiments. ${ }^{*} \mathrm{P}<0.05$ and $* * P$ $<0.01$ vs. Sham, ${ }^{\#} \mathrm{P}<0.05$ vs. BDL. (B) Protein levels were measured by immunoblotting with antibodies against GRP78, IRE1, ATF4, CHOP and $\beta$-actin. BDL: bile duct ligation. plasia followed by bile duct obstruction (19). However, the physiological relevance is controversy between two experimental models. It has been reported that metformin attenuated oxidative stress and liver damage after BDL in rats suggesting the protective role of metformin in cholestasis (20). Lien and colleagues showed that metformin aggravated intrahepatic cholestasis induced by ANIT (21). Interestingly Lien and colleagues showed that metformin inhibited plasma levels of ALT and AST induced by food intake supplemented with $0.5 \%$ TCA bile acids and reduced bile acid excretion in vivo. These results suggested that metformin selectively inhibited bile acid-induced hepatic responses. In the current study, we found that metformin inhibited BDL-induced liver injury, inflammation, and ER stress. These discrepancies remain to be determined by further studies under similar experimental conditions.

\section{Metformin inhibits GCDCA-induced UPR and cell death in primary hepatocytes}

In vivo experiments confirmed that ER stress, inflammatory response, and chemokine expression increase after BDL. Based on these results, isolated primary hepatocytes from C57BL/6
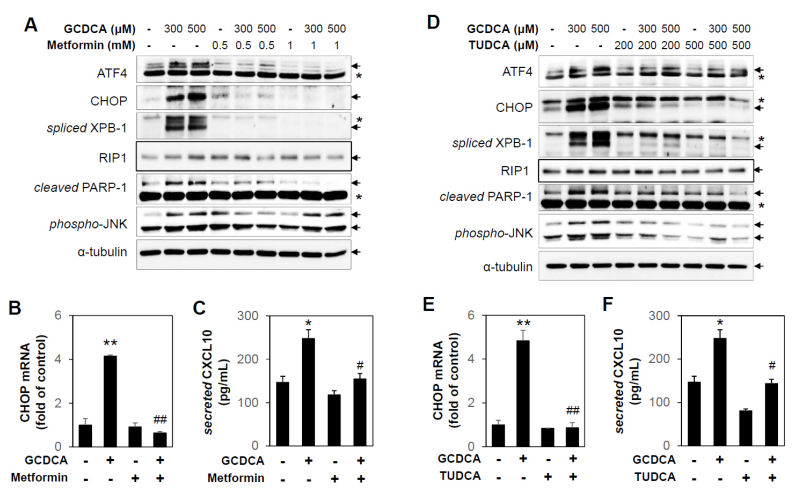

Fig. 4. Metformin and TUDCA inhibit GCDCA-induced UPR and cell death in primary hepatocytes. (A) $\mathrm{C} 57 \mathrm{BL} / 6$ primary hepatocytes were pretreated with metformin $(0.5$ or $1 \mathrm{mM})$ for $1 \mathrm{~h}$ and then incubated with GCDCA $(300$ or $500 \mu \mathrm{M})$ for $6 \mathrm{~h}$. Protein levels were measured by immunoblotting with antibodies against ATF4, CHOP, spliced XBP-1, Rip1, cleaved PARP-1, phospho-JNK, and $\alpha$-tubulin. Asterisks indicate non-specific bands. (B, E) C57BL/6 primary hepatocytes were pretreated with metformin $(0.5 \mathrm{mM})$ or TUDCA $(200 \mu \mathrm{M})$ for $1 \mathrm{~h}$ and then incubated with GCDCA $(300 \mu \mathrm{M})$ for $6 \mathrm{~h}$. The CHOP mRNA levels were measured by performing qRT-PCR. Relative expression levels were normalized to GAPDH. Results are expressed as mean \pm SD values and are representative of three independent experiments. **P $<0.01$ vs. vehicle, ${ }^{\# \#} \mathrm{P}<0.01$ vs. GCDCA. (C, F) The secreted amounts of CXCL10 in conditioned medium were determined by ELISA assay. Results are expressed as mean \pm SE values and are representative of three independent experiments. ${ }^{*} P<0.05$ vs. vehicle, ${ }^{\#} \mathrm{P}<0.05$ vs. GCDCA. (D) C57BL/6 primary hepatocytes were pretreated with TUDCA $(200$ or $500 \mu \mathrm{M})$ for $1 \mathrm{~h}$ and then incubated with GCDCA $(300$ or $500 \mu \mathrm{M})$ for $6 \mathrm{~h}$. Protein levels were measured by immunoblotting with antibodies against ATF4, CHOP, spliced XBP-1, Rip1, cleaved PARP-1, phospho-JNK, and $\alpha$-tubulin. Asterisks indicate non-specific bands. 
mice were treated with glycochenodeoxycholic acid (GCDCA), one of the bile acids. Protein expressions of ATF4, CHOP, spliced XBP-1, and mRNA levels of CHOP were increased by GCDCA treatment, suggesting that GCDCA activates the UPR signaling pathway (Fig. 4A and 4B). In addition, the necroptosis marker protein Rip1 and the apoptosis marker protein cleaved PARP-1 were increased by GCDCA treatment, and these inductions were inhibited by metformin treatment (Fig. 4A). Furthermore, the inflammation marker protein phospho-JNK and secreted CXCL10 in conditioned medium were increased by GCDCA treatment, and these inductions were inhibited by metformin treatment (Fig. 4A and $4 \mathrm{C}$ ). Therefore, the responses induced by bile acid are thought to be related to ER stress. It has been reported that ASK1 deficiency has a critical role in ameliorating cholestatic liver injury via inhibition of inflammation, necrosis, and proliferation (22). Although we observed that metformin inhibited GCDCA-induced activation of JNK, which is downstream of the ASK1 pathway in primary hepatocytes, the underlying mechanisms of metformin in cholestasis models remains to be determined.

We next sought to evaluate the role of UPR in primary hepatocytes exposed to GCDCA. The mouse primary hepatocytes were pretreated by tauroursodeoxycholic acid (TUDCA), an ER stress inhibitor, and, as expected, induction of ATF4, $\mathrm{CHOP}$, and spliced XBP-1 protein levels and CHOP mRNA levels were significantly inhibited by TUDCA (Fig. 4D and 4E). In addition, necroptosis, apoptosis and inflammation marker proteins induced by GCDCA were inhibited by TUDCA (Fig. $4 \mathrm{D}$ and $4 \mathrm{~F})$. These results suggest that inhibition of UPR is responsible for the protective role of metformin against the bile acid-induced damage response in vitro.

In summary, our results demonstrate that metformin can reduce liver injury caused by the stagnation of bile by reducing neutrophil infiltration and inflammatory mediators. In addition, metformin protects hepatocytes against bile acid-induced inflammation and apoptosis through the inhibition of ER stress.

\section{MATERIALS AND METHODS}

\section{Reagents and antibodies}

Metformin, GCDCA, and TUDCA were obtained from SigmaAldrich (St. Louis, MO, USA). The following antibodies were purchased from a variety of vendors: KDEL (GRP94, GRP78) (Enzo Life Sciences, Lörrach, Germany), GADD153 (CHOP), CREB-2 (ATF4) (Santa Cruz, Santa Cruz, CA, USA), spliced XBP-1 (BioLegend, San Diego, CA, USA); IRE1 $\alpha$, phospho-JNK, PARP-1 (Cell signaling Technology, Danvers, MA, USA); Cyclooxynease-2 (Cayman Chemical, Ann Arbor, MI, USA); $\beta$-actin and $\alpha$-tubulin (Sigma-Aldrich).

\section{Isolation of primary hepatocyte}

Primary hepatocytes were obtained from C57BL/6 mice (8-10 weeks old, Central Lab Animal Inc., Seoul, Korea). Initially, the mice were sacrificed and the peritoneal cavity was opened.
HBSS was injected into the portal vein at a low flow rate (7-9 $\mathrm{ml} / \mathrm{min}$ ), and the inferior vena cava was cut and perfused for 20 min. Digestion medium [Low glucose-DMEM (Gibco, Grand Island, NY, USA) supplemented with $0.03 \%$ collagenase (Worthington, Lakewood, NJ, USA), 15 mM HEPES, $50 \mathrm{U} / \mathrm{ml}$ penicillin, and $50 \mu \mathrm{g} / \mathrm{ml}$ streptomycin] was injected after perfusion. Livers were isolated from each mouse. Hepatocytes were harvested by using forceps in isolation medium [High glucose-DMEM (HyClone, Logan, UT, USA) supplemented with $1 \mathrm{mM}$ L-glutamine, $15 \mathrm{mM}$ HEPES, $100 \mathrm{nM}$ dexamethasone, $50 \mathrm{U} / \mathrm{ml}$ penicillin, and $50 \mu \mathrm{g} / \mathrm{ml}$ streptomycin]. The cell suspension was filtered through a $70 \mu \mathrm{m}$ strainer and then centrifuged at $250 \mathrm{r} / \mathrm{min}$ for $4 \mathrm{~min}$. The supernatant was removed and washed with fresh isolation medium. This process was repeated two more times. Isolated hepatocytes were seeded at equal densities and incubated in isolation medium for $1 \mathrm{~h}$, and then changed to culture medium [Low glucoseDMEM supplemented with $10 \%$ FBS, $2 \mathrm{mM}$ L-glutamine, 5 $\mathrm{mM}$ HEPES, $10 \mathrm{nM}$ dexamethasone, $50 \mathrm{U} / \mathrm{ml}$ penicillin and 50 $\mu \mathrm{g} / \mathrm{ml}$ streptomycin]. Cells were incubated in a humidified $5 \%$ $\mathrm{CO}_{2} / 95 \%$ air atmosphere at $37^{\circ} \mathrm{C}$. Secreted amounts of CXCL10 in conditioned medium were determined by ELISA kit, according to the manufacturer's instructions.

\section{Western blot analysis}

Tissue and cell samples were lysed in radioimmunoprecipitation assay (RIPA) lysis buffer supplemented with $1 \mathrm{mM}$ PMSF and $0.01 \mathrm{mM}$ protease inhibitor cocktail. Lysates were incubated on ice for $15 \mathrm{~min}$ and centrifuged at 15,000 $\times \mathrm{g}$ for $10 \mathrm{~min}$ at $4^{\circ} \mathrm{C}$. Protein concentrations were determined using the Bradford assay. Proteins were separated by SDS-PAGE and transferred to polyvinylidene difluoride (PVDF) membranes, which were immunoblotted with the indicated primary antibodies and then with the corresponding secondary antibodies. Signals were visualized using electrochemiluminescence detection reagents (Millipore, Billerica, MA, USA) according to the manufacturer's instructions.

\section{Quantitative real-time RT-PCR}

The mRNA levels were determined by performing quantitative real-time RT-PCR (qRT-PCR). Briefly, total RNA was isolated using TRIzol ${ }^{\mathbb{R}}$ Reagent (Invitrogen, Carlsbad, CA, USA), and the reverse transcription reaction was conducted using TaqMan reverse transcription reagents (Applied Biosystems, Carlsbad, CA, USA), according to the manufacturer's instructions. The qRT-PCR was conducted with $1 \mu \mathrm{l}$ of template cDNA and Power SYBR Green (Applied Biosystems) in an ABI PRISM 7500 unit (Applied Biosystems). Quantification was carried out using the efficiency-corrected $\Delta \Delta \mathrm{Cq}$ method. The primers used to amplify DNA sequences were as follows: mouse MLKL forward: 5'-GGATTGCCCTGAGTTGTTGC-3' and reverse: 5'-AA CCGCAGACAGTCTCTCCA-3'; mouse Rip1 forward: 5'-GCCC AACCG CGCTGAGTACA-3' and reverse: $5^{\prime}$-TGCCTTCTATG GCCTCCACGA-3'; mouse Rip3 forward: 5'-TGTCAAGTTATG 
GCCTACTGGTGCG-3' and reverse: 5'-AACCATAGCCTT CAC CTCCCAGGAT-3'; mouse CXCL10 forward: 5'-AGAACGGTG CGCTGCAC-3' and reverse: 5'-CCTATGGCCCTGGGTCTCA-3'; mouse CXCL2 forward: 5'-CGCTGTCAATG CCTGAAGAC-3' and reverse: 5'-ACACTCAAGCTCTGGATGTTCTTG-3'; mouse CHOP forward: 5'-GCATGAAGGAGAAGGAGCAG-3' and reverse: 5'-CTTCCGGAGAGACAGA CAGG-3'; mouse ATF4 forward: 5'-AGAGCTCATCTGGCATGGTT-3' and reverse: 5'-TCG ATGCTCTGTTTCGAATG-3'; mouse spliced XBP-1 forward: 5'GTGTCAGAGTCCATGGGA-3' and reverse: 5'-GAGTCCGCA GCAGGTG-3'; mouse IL-6 forward: 5'-TCAGAATTGCCAT TG CACA-3' and reverse: 5'-GTCGGAGGCTTAATTACACATG-3'; mouse TNF- $\alpha$ forward: $5^{\prime}$-AGACACGGCTGAGCATACAT- $3^{\prime}$ and reverse: 5'-GCTCAGGAGGTCAGCAACA-3'; mouse GAPDH forward: 5'-GTGATG GCATGGACTGTGGT-3' and reverse: 5'GGAGCCA AAAGGGTCATCAT-3'.

\section{Animal experiments}

Under anesthesia, the abdominal cavity of specific pathogenfree male C57BL/6 mice (8-10 weeks old) was opened and the common bile duct was ligated twice with 5-0 surgical silk, and the bile duct was cut between the ligatures. Sham operations were performed similarly, except for performing ligation and transection of the bile duct. Metformin was injected either i.p. or oral gavage at $200 \mathrm{mg} / \mathrm{kg} /$ day. Serum and livers (gallbladder removed) were collected 3 days after BDL. Plasma levels of TNF- $\alpha$ were determined by ELISA kit, according to the manufacturer's instructions. All animal experiments were conducted in accordance with a protocol approved beforehand by the Institutional Animal Care and Use Committee of Yeungnam University College of Medicine, Daegu, Republic of Korea. In addition to this, all experiments were performed in accordance with the relevant guidelines and regulations.

\section{Histological analyses}

Liver tissues were fixed in $10 \%$ formalin, embedded in paraffin, and then cut into $5 \mu \mathrm{m}$ thick slices. Sections were subjected to hematoxylin and eosin ( $\mathrm{H} \& \mathrm{E})$ staining and immunohistochemistry $(\mathrm{IHC})$ analysis. The $\mathrm{IHC}$ analysis was conducted using a rabbit specific HRP/DAB (ABC) Detection IHC kit (Abcam, Cambridge, UK), according to the manufacturer's instructions. All images were collected using an optical microscope (Nikon, Tokyo, Japan).

\section{Statistics}

Results in bar graphs are expressed as mean \pm SD or \pm SE values for three independent experiments. Statistical significance was assessed by Student's $t$-test or by ANOVA followed by the Bonferroni post hoc test for multiple group comparisons using GraphPad Prism 5.0 (Graph-Pad Software Inc.). Probability values ( $P$ values) of $<0.05$ were considered statistically significant.

\section{ACKNOWLEDGEMENTS}

This research was supported by the Medical Research Center Program (2015R1A5A2009124) and the Basic Science Research Program (2018R1A2B6004664) through the Korean National Research Foundation funded by the Ministry of Science, ICT, and Future Planning.

\section{CONFLICTS OF INTEREST}

The authors have no conflicting interests.

\section{REFERENCES}

1. Bailey CJ and Turner RC (1996) Metformin. N Engl J Med 334, 574-579

2. Rena G, Pearson ER and Sakamoto K (2013) Molecular mechanism of action of metformin: old or new insights? Diabetologia 56, 1898-1906

3. Kim J, Kwak HJ, Cha JY et al (2014) Metformin suppresses lipopolysaccharide (LPS)-induced inflammatory response in murine macrophages via activating transcription factor3 (ATF-3) induction. J Biol Chem 289, 23246-23255

4. Dai J, Liu M, Ai Q et al (2014) Involvement of catalase in the protective benefits of metformin in mice with oxidative liver injury. Chem Biol Interact 216, 34-42

5. Wheaton WW, Weinberg SE, Hamanaka RB et al (2014) Metformin inhibits mitochondrial complex I of cancer cells to reduce tumorigenesis. Elife 3, e02242

6. Jung TW, Lee MW, Lee YJ and Kim SM (2012) Metformin prevents endoplasmic reticulum stress-induced apoptosis through AMPK-PI3K-c-Jun NH2 pathway. Biochem Biophys Res Commun 417, 147-152

7. Trauner M, Meier PJ and Boyer JL (1998) Molecular pathogenesis of cholestasis. N Engl J Med 339, 1217-1227

8. Kaplowitz N (2004) Drug-induced liver injury. Clin Infect Dis 38 Suppl 2, S44-S48

9. Cai SY, Ouyang X, Chen Y et al (2017) Bile acids initiate cholestatic liver injury by triggering a hepatocyte-specific inflammatory response. JCl Insight 2, e90780

10. Tamaki N, Hatano E, Taura K et al (2008) CHOP deficiency attenuates cholestasis-induced liver fibrosis by reduction of hepatocyte injury. Am J Physiol Gastrointest Liver Physiol 294, G498-505

11. Gujral JS, Farhood A, Bajt ML and Jaeschke H (2003) Neutrophils aggravate acute liver injury during obstructive cholestasis in bile duct-ligated mice. Hepatology 38, 355363

12. Schroder M and Kaufman RJ (2005) ER stress and the unfolded protein response. Mutat Res 569, 29-63

13. Lee DH, Lee B, Park JS et al (2019) Inactivation of Sirtuin2 protects mice from acetaminophen-induced liver injury: possible involvement of ER stress and S6K1 activation. BMB Rep 52, 190-195

14. Kwon MY, Hwanf N, Lee SJ and Chung SW (2019) Nucleotide-binding oligomerization domain protein 2 attenuates ER stress-induced cell death in vascular smooth muscle cells. BMB Rep 52, 665-670 
15. Woudenberg-Vrenken TE, Conde de la Rosa L, BuistHoman M, Faber KN and Moshage H (2013) Metformin protects rat hepatocytes against bile acid-induced apoptosis. PLoS One 8, e71773

16. Tag CG, Sauer-Lehnen S, Weiskirchen S et al (2015) Bile duct ligation in mice: induction of inflammatory liver injury and fibrosis by obstructive cholestasis. J Vis Exp 96, e52438

17. Georgiev P, Jochum W, Heinrich S et al (2008) Characterization of time-related changes after experimental bile duct ligation. Br J Surg 95, 646-656

18. Afonso MB, Rodrigues PM, Simao AL et al (2016) Activation of necroptosis in human and experimental cholestasis. Cell Death Dis 7, e2390
19. Kossor DC, Goldstein RS, Ngo W et al (1995) Biliary epithelial cell proliferation following alpha-naphthylisothiocyanate (ANIT) treatment: relationship to bile duct obstruction. Fundam Appl Toxicol 26, 51-62

20. Sadeghi H, Jahanbazi F, Sadeghi H et al (2019) Metformin attenuates oxidative stress and liver damage after bile duct ligation in rats. Res Pharm Sci 14, 122-129

21. Lien F, Berthier A, Bouchaert E et al (2014) Metformin interferes with bile acid homeostasis through AMPK-FXR crosstalk. J Clin Invest 124, 1037-1051

22. Noguchi H, Yamada S, Nabeshima A et al (2014) Depletion of apoptosis signal-regulating kinase 1 prevents bile duct ligation-induced necroinflammation and subsequent peribiliary fibrosis. Am J Pathol 184, 644-661 